\title{
Transatlantica
}

Revue d'études américaines. American Studies Journal

\section{Collin Meissner. Henry James and the Language of} Experience.

Cambridge : Cambridge UP, 1999. ix+237p. £37.50, \$59.95.

\section{Annick Dupperay}

\section{(2) OpenEdition}

\section{Journals}

Édition électronique

URL : http://journals.openedition.org/transatlantica/683

DOI : $10.4000 /$ transatlantica.683

ISSN : 1765-2766

Éditeur

AFEA

Référence électronique

Annick Dupperay, "Collin Meissner. Henry James and the Language of Experience. », Transatlantica [En ligne], 1 | 2003, mis en ligne le 05 avril 2006, consulté le 29 avril 2021. URL : http://

journals.openedition.org/transatlantica/683 ; DOI : https://doi.org/10.4000/transatlantica.683

Ce document a été généré automatiquement le 29 avril 2021.

\section{c) (i) $९$}

Transatlantica - Revue d'études américaines est mis à disposition selon les termes de la licence Creative Commons Attribution - Pas d'Utilisation Commerciale - Pas de Modification 4.0 International. 


\section{Collin Meissner. Henry James and the Language of Experience.}

Cambridge : Cambridge UP, 1999. ix+237p. £37.50, \$59.95.

\section{Annick Dupperay}

1 Conformément aux développements récents de la critique jamesiennne, qui s'efforce de réconcilier formalisme et historicisme, Meissner redécouvre ce qu'il est convenu d'appeler la dimension "politique » de l'oeuvre de James et insiste sur la fonction «civique» du récit de fiction telle que l'envisageait le romancier lui-même en définissant "the civic use of imagination" (voir la préface à "The Lesson of the Master »). Meissner procède à une analyse textuelle approfondie de trois romans représentatifs des étapes successives de l'oeuvre fictionnelle-The American, The Portrait of a Lady et The Ambassadors - qu'il rapproche en fin d'ouvrage des trois volumes de l'autobiographie. S'il s'agit de démontrer l'interrelation entre esthétique et politique, l'auteur souhaite cependant s'inscrire en porte-à-faux par rapport aux approches «new historicist» comme celles de Mark Seltzer (Henry James and the Art of Power, 1984). Seltzer perçoit chez James une double stratégie selon laquelle l'écrivain déplore et reconnait à la fois la collusion entre l'art et le pouvoir et ce faisant, révèle son rapport de connivence avec l'ordre établi et le discours du maitre. Meissner, quant à lui, associe l'engagement "politique » de l'écriture jamesienne à son pouvoir de subversion et de contestation des canons culturels et sociaux, même s'il insiste sur le caractère essentiellement individualiste de la démarche. Malgré ses affinités avec Walter Pater, poursuit Meissner, James entra très tôt en guerre - dès 1875 avec Roderick Hudson et 1877 avec The American - contre les méfaits de l'esthétisme : " the immoral underside of aestheticism, at least in so far as the aesthete cultivated a hypersensual response to life ».

2 La complexité des relations humaines en sa dimension sociale fait elle aussi partie de ce réel que l'on ne peut manquer de rencontrer tôt ou tard (voir préface à The American). La notion d'expérience s'envisage suivant deux logiques contradictoires qui s'affrontent dès The American; on discerne une logique accumulatrice et empirique, un mode de consommation, par lequel la dite expérience vient renforcer les structures 
mentales et schémas culturels préétablis. Elle entre en conflit avec une autre vision de l'expérience, sous le signe de l'altérité et de l'altération, une démarche négatrice - «transformative and non-affirmative ». L'exemple par excellence de cette rencontre déstabilisatrice avec l'autre que soi est la scène du Lambinet (The Ambassadors) : "The sudden explosion of Strether's frame of reference matches exactly James's understanding of reality as an unfixable, ever-expanding horizon, what William James referred to as a 'multiverse' ». La rencontre de l'inconnu ou de l'étrange devient mode de connaissance et l'on appréciera de ce point de vue d'intéressants parallèles entre The Portrait of a Lady (Isabel's « midnight vigil »), The Ambassadors et The American. Ce dernier, souvent perçu à tort comme un romance manqué («a failed comic romance ») entraîne déjà personnage et lecteurs, par cercles concentriques, vers une forme d'épiphanie négative que Meissner appelle «an interpretive quandary »: «the failure to understand often becomes the medium of understanding ». Le problème étant que, vu l'extrême fluidité de leur expérience, les personnages ne peuvent plus être signifiés par l'intrigue qui les contient; James les laisse "en l'air» (voir Notebooks, Portrait of a Lady). D'où l'énigmaticité des dénouements (Portrait et The Ambassadors), et la frénésie interprétative de plusieurs générations de lecteurs contraints d'aller au-delà du texte de fiction, d'envisager la multiplicité des possibles - l'avenir extra-textuel des héros. C'est ainsi, selon Meissner, que James maintient le dialogue entre l'art et la vie et rappelle les limites du formalisme esthétisant.

On appréciera la réévalution des dénouements les plus équivoques, ainsi que le parallèle établi entre les représentations fictionnelles de la conscience artistique et leur contrepartie autobiographique. L'autobiographie de James se situe à l'intersection entre l'art et la vie; la figure de l'auteur se libère à son tour du cadre formel de la narration puisque le dernier volume demeura inachevé. Tout n'est pas nouveau dans l'ouvrage de Meissner, mais l'analyse est solide, souvent convaincante et remarquablement rédigée.

\section{INDEX}

Thèmes : Recensions

\section{AUTEUR}

\section{ANNICK DUPPERAY}

Université de Provence 